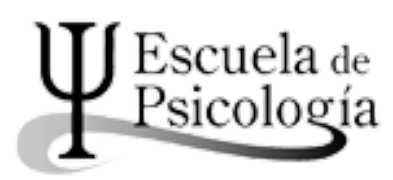

Wímblu, Rev. estudiantes Esc. de psicología, Univ. de Costa Rica. 12 (1): 57-67, 2017 / ISSN: 1659-2107

\title{
Efecto de la experiencia corporal de frío sobre la calidez interpersonal hacia grupos sociales. Un estudio desde la cognición corporizada ${ }^{1}$
}

\author{
Effect of experience cold on interpersonal warmth toward social groups. \\ A study from the embodied cognition
}

Fiorella Jara Sanabria

\begin{abstract}
Resumen. Para la cognición corporizada el sistema sensoriomotor es parte constitutiva de los procesos cognitivos, por ello se enfoca en la relación entre cuerpo físico y las capacidades perceptivas. Con el propósito de incrementar la evidencia de este tema en Costa Rica, se desarrolló un experimento para establecer el efecto de la experiencia corporal de frío sobre la actitud hacia grupos sociales en estudiantes universitarios. La manipulación experimental se realizó mediante el ajuste a $19,5 \mathrm{C}^{\circ}$ del aire acondicionado de la sala del grupo experimental. La muestra la conformaron 40 estudiantes de la Universidad de Costa Rica (20 en cada grupo) quienes contestaron un cuestionario con dos escalas. Los resultados no mostraron diferencias significativas entre grupos, lo cual permite rechazar la hipótesis planteada. Sin embargo, la calificación general de los participantes, mostró una actitud desfavorable hacia las personas homosexuales, en comparación con las heterosexuales, siendo estadísticamente significativa.
\end{abstract}

Palabras clave: Cognición corporizada, calidez interpersonal, grupos sociales, homofobia.

Abstract. For the embodied cognition the sensorimotor system is a constitutive part of the cognitive processes, that why it focuses on the relationship between physical body and perceptive capabilities. In order to increase the evidence of this in Costa Rica, an experiment was developed to establish the effect of cold on the embodied cognition on attitudes towards social groups of univerisity students. The experimental manipulation was done through the adjustment of the air conditioning temperature to $19,5 \mathrm{C}^{\circ}$ for the experimental group. The sample consisted of 40 Universidad de Costa Rica students (20 in each group) which answered to a questionaire with two scales. The results did not show any significant differente between the groups, which rejects the original hypothesis. Nevertheless, the general participants score, showed a negative attittude towards homosexual people, compared with heterosexual, being statistically significant.

Key Words: Embodiment, interpersonal warmth, social groups, homophobia.

* Universidad de Costa Rica. Costa Rica. Correo electrónico: fiojs@hotmail.com

** Recepción: 6/11/2016 Aceptación: 6/2/2017 


\section{Introducción}

Tradicionalmente, la cognición ha sido descrita como un fenómeno individual dirigido por el sistema nervioso, en el cual, se manipulan las representaciones mentales que se tienen de los objetos o personas (Encabo, 2005, Soto, 2007); su estudio se ha enfocado en las redes neuronales, modelos computacionales y las bases biológicas de la actividad cerebral (Wilson \& Foglia, 2016).

Sin embargo, entre la década de los años ochenta y noventa del siglo pasado surgió un enfoque alternativo de investigación conocido como embodiment o cognición corporizada. La cual parte del supuesto, de que el sistema sensoriomotor es parte constitutiva de los procesos cognitivos (Letheby, 2012).

El principal objetivo del embodiment es comprender cómo las características del cuerpo físico influyen en las capacidades perceptivas, ofreciendo una perspectiva amplia e integradora de la cognición (Rodríguez, 2012, Wilson \& Foglia, 2016). Para Wilson \& Foglia (2016) un ejemplo simple de ello, son los conceptos de arriba-abajo o izquierda-derecha, los cuales no podrían definirse sin el posicionamiento corporal.

En el mismo plano, estudios dirigidos por Beirute-Herrera, Angulo-Castro, Fallas-Gamboa \& Prado-Calderón (2014) y Inbar, Pizarro \& Bloom (2012) evidenciaron cómo la presencia de un olor ambiental desagradable influyó negativamente en las actitudes hacia personas homosexuales. Y el de Bargh \& Shalev (2012) y Williams \& Bargh (2008) demostraron cómo la experiencia corporal de frío y calor, respectivamente, intervinieron en la formación de impresiones de la personalidad de los participantes del grupo experimental.

Tomando como principales antecedentes las dos últimas investigaciones y con el fin de incrementar la evidencia del embodiment en el país se desarrolló un estudio experimental que se propuso establecer el efecto de la experiencia corporal de frío sobre la actitud hacia grupos sociales en estudiantes universitarios.

Basado en William \& Bargh (2008), la hipótesis alternativa consistió en que las personas expuestas a un ambiente frío tuviesen actitudes más desfavorables hacia los grupos sociales en comparación con las del grupo control; por medio de una manipulación experimental no prevista ni desagradable para los participantes. La variable dependiente se midió mediante los resultados de dos escalas que evaluaban la formación de impresiones de la personalidad (Asch, 1952) y la actitud hacia grupos sociales (Inbar et al. 2012) respectivamente.

\section{Metodología}

\section{Enfoque y método de investigación}

El abordaje de investigación se efectuó desde el enfoque cuantitativo y el método experimental. El diseño fue unifactorial de bloques aleatorizados, que se caracteriza por medir el efecto de una sola variable de tratamiento sobre una muestra que posee la misma cantidad de participantes para ambas condiciones (Corbeta, 2001; Fontes et al., 2015). En este ensayo, la variable independiente fue la temperatura corporal y la dependiente la calidez interpersonal. Y corresponde a una réplica del estudio de Williams \& Bargh (2008).

\section{Población y muestra}

La población meta del estudio fueron los estudiantes universitarios de la Sede Rodrigo Facio de la Universidad de Costa Rica, quienes debían estar matriculados durante el ciclo II del 2015 como requisito de inclusión. La muestra fue de tipo no probabilística (Creswell, 2012) y las personas que accedieron a colaborar se asignaron a los grupos aleatoriamente mediante el software Research Randomizer.

Esta estuvo conformada por 20 mujeres y 20 hombres entre los 17 y 25 años de edad, con una media de 20,28 años $(S D=2,025)$. En cada condición hubo una distribución equivalente de los participantes (20 personas por grupo), aunque no fue así en razón de sexo en donde el grupo experimental tuvo $65 \%$ de hombres y el $35 \%$ de mujeres; y el control $65 \%$ de mujeres y $35 \%$ de hombres.

El $87,5 \%$ de los participantes se encontraba empadronado en alguna carrera de la Facultad de Ciencias Sociales, entre las que destacan Psicología con un $35 \%$ del total y Ciencias Políticas con $27,5 \%$. Entre los restantes, el $7,5 \%$ eran estudiantes de la Facultad de Ingeniería y el 5\% de la Facultad de Farmacia.

El lugar de residencia de los participantes era variado, pero predominaron quienes habitaban los cantones de Desamparados y San José (lugares que representaron un $10 \%$ cada uno del total de la muestra). Además, el $82,5 \%$ de los jóvenes refirieron que su ocupación era estudiante y el 97,5\% de los participantes calificaron su grupo étnico como blanco o mestizo. Finalmente, el estado civil de todos era la soltería.

\section{Materiales}

Como instrumento de recolección de la información se empleó un cuestionario que contenía 4 secciones. La primera solicitaba información sociodemográficas, como lugar de procedencia, sexo, estado civil, carrera empadronada, edad, grupo étnico de referencia y ocupación. La segunda era una escala de diferencial semántico (Corbetta, 2001) Ilamada Escala de Formación de Impresiones (Forming Impressions of Personality) (Asch, 1952), en la cual se clasifica del 0 al 100 a una persona prototipo, mediante un conjunto de antónimos. Williams \& Bargh (2008) también reportaron su uso.

El siguiente apartado también correspondía a una escala, el Termómetro de sentimientos (Inbar et al., 2012). En ella se cataloga la percepción hacia 11 
grupos sociales (personas indígenas, chinas, colombianas, ateas, obesas, pobres, campesinas, adultas mayores, católicas, nicaragüenses y afrocostarricenses) del 0 al 100 (donde 0 es muy desfavorable y 100 muy favorable).

La última parte, eran 8 preguntas que buscaban determinar si el participante sospechaba que la manipulación de la temperatura era parte del estudio. Por ello, se les solicitaba que concretarán la hipótesis de la investigación, que mencionaran los factores que hubiesen interferido durante la administración del instrumento y algunas otras variables de confusión como el número de individuos que lo acompañaron al realizar la tarea.

\section{Procedimiento de recolección de datos}

El experimento se realizó en diferentes espacios del Edificio de la Facultad de Ciencias Sociales (las cuales se solicitaron formalmente previo a la recogida de información) y fue presentado a las personas como: Actitudes de estudiantes universitarios hacia grupos sociales en el contexto costarricense.

Durante 2 semanas del mes de octubre entre las $2 \mathrm{pm}$ y $4 \mathrm{pm}, 3$ investigadores se encargaron de invitar a estudiantes que se encontraran en los pasillos, soda y salas de estudio a participar, asimismo, algunas docentes permitieron externar la propuesta a sus alumnos en horario de clase. En este primer acercamiento, se ofrecía una explicación verbal sobre los propósitos de la investigación, las actividades que debían realizar y duración de la participación.

A las personas que accedían se les entregaba una copia del consentimiento informado, elaborado bajo los parámetros del Comité Ético-Científico de la Universidad de Costa Rica; y se les asignaban aleatoriamente al grupo control o experimental.

Los participantes del grupo control se dirigían a un aula de clase o sala de reuniones (según disponibilidad), mientras que en todas las ocasiones, los del grupo experimental se condujeron a un aula del Instituto de Investigaciones Psicológicas (IIP). La manipulación experimental, se realizó mediante el aire acondicionado, que se ajustó 20 minutos antes de atender a los participantes a 19,5 $\mathrm{C}^{\circ}$ de temperatura. En el aula del grupo control no se manipuló la temperatura.

Antes de entregar los cuestionarios los investigadores resaltaban aspectos clave del estudio y ofrecían las instrucciones. Una de esas indicaciones era mantenerse en el aula hasta que todos los participantes hubiesen finalizado, con el propósito de no alterar la temperatura ambiental y poder ofrecer de forma unánime la explicación sobre la verdadera conjetura de la investigación.

Una vez que concluía la última persona, se recogían todos los instrumentos y se proporcionaba una explicación sobre el experimento, los supuestos teóricos del embodied cognition y la hipótesis del estudio. Al finalizar, se les ofrecía un espacio para resolver dudas o brindar comentarios, se verificaba verbalmente que todos se sintiesen a gusto y se les agradecía la participación. En todas las aplicaciones del instrumento, los 3 investigadores rotaron funciones con el fin de evitar convertirse en variables de confusión.

\section{Análisis de datos}

El análisis de datos se efectuó con el programa IBM SPSS Statistics versión 2.0 para MacOS. Para evaluar la normalidad de los datos se empleó la prueba Kolmogorov-Smirnov (K-S) y se utilizaron las pruebas a) t de Student para muestras relacionadas $(\mathrm{t})$ y $\mathrm{b}$ ) chi-cuadrado $\left(\mathrm{x}^{2}\right)$, para comparar las medias de los grupos y someter a prueba la hipótesis.

\section{Resultados}

Primeramente, se encontraron diferencias significativas $(t(38)=3,075$, $p=0,004)$ en la percepción térmica entre grupos, en donde el grupo control evaluó el ambiente como poco frío mientras que el experimental lo calificó como bastante frío. Hecho que verifica que la manipulación de la variable independiente fue exitosa.

En cuanto al desagrado provocado por los cambios de temperatura, el promedio de clasificación en una escala de 1 a 10 fue $X=2,38, S D=2,39$ lo cual evidencia que el frío no fue un factor desagradable para los participantes y de haber resultados significativos, estas diferencias deberían ser atribuidas a la temperatura y no a la aversión o incomodidad con la sala en la que se encontraban durante el experimento.

Además, el promedio de interferencia de factores ambientales fue de $X=1,83, S D=2,65$ en una escala del 1 al 10; $y$ entre los elementos mencionados se incluyó el ruido y la temperatura con $15 \%$ cada uno. Aun así, ningún participante consideró a la temperatura dentro de la posible hipótesis de investigación y el $90 \%$ de las personas negaron haber sospechado que era parte del estudio; aunque todos los casos positivos se ubicaron en el grupo experimental, sin que la diferencia fuese significativa. Asimismo, al contrastar las edades y el sexo de los participantes entre grupos, no se obtuvieron diferencias significativas lo cual corrobora la posibilidad de comparación.

Por otra parte, tanto los resultados de formación de impresiones de la personalidad, reunidos en la Tabla 1, como los de actitudes hacia grupos sociales, en la Tabla 2, no mostraron diferencias significativas entre grupos, por lo cual se rechaza la hipótesis alternativa planteada. Incluso la calificación total de la escala de actitudes hacia grupos sociales (calidez general) de las personas de la condición experimental fue mayor $(X=82,07, S D=13,92)$ en comparación con el 
grupo control ( $X=78,87, S D=14,87)$, sin presentar significancia $(p=0,49)$.

No obstante, se efectuaron otros análisis orientados a determinar qué colectivo había sido mejor y peor clasificado por la totalidad de la muestra. Lo cual evidenció que la actitud más favorable de los participantes fue hacia mujeres heterosexuales; y la menos favorable hacia las personas católicas, ateas y las mujeres y hombres homosexuales.

TABLA 1.

Formación de impresiones de la personalidad según grupo de tratamiento

\begin{tabular}{lccccc}
\hline \multicolumn{1}{c}{ IMPRESIONES } & \multicolumn{4}{c}{ GRUPO CONTROL } & \multicolumn{3}{c}{ GRUPO EXPERIMENTAL } \\
& $\mathrm{M}$ & $\mathrm{DE}$ & $\mathrm{M}$ & $\mathrm{DE}$ & $\mathrm{t}$ \\
Generosa & 56,32 & 19,01 & 61,36 & 22,12 & $-0,77$ \\
Prudente & 79,90 & 17,20 & 77,04 & 21,77 & 0,46 \\
Feliz & 60,71 & 15,29 & 59,66 & 15,93 & 0,21 \\
Amena & 56,54 & 19,15 & 59,58 & 16,87 & $-0,53$ \\
Divertida & 46,95 & 11,64 & 53,65 & 14,77 & $-1,60$ \\
Sociable & 54,77 & 19,27 & 62,18 & 17,59 & $-1,27$ \\
Popular & 54,60 & 13,43 & 55,72 & 17,80 & $-0,22$ \\
Confiable & 73,03 & 21,06 & 76,31 & 17,86 & $-0,53$ \\
Importante & 72,51 & 18,75 & 68,41 & 18,85 & 0,69 \\
Compasiva & 59,38 & 18,08 & 60,87 & 24,66 & $-0,22$ \\
Bella & 53,41 & 14,07 & 56,91 & 16,29 & $-0,73$ \\
Perseverante & 80,94 & 18,37 & 79,92 & 23,00 & 0,16 \\
Solidaria & 60,44 & 16,54 & 64,59 & 22,00 & $-0,68$ \\
Minuciosa & 73,79 & 23,04 & 77,62 & 15,50 & $-0,62$ \\
Habladora & 49,24 & 20,63 & 52,15 & 17,12 & $-0,48$ \\
Fuerte & 65,94 & 18,62 & 65,41 & 20,05 & 0,09 \\
Honesta & 72,58 & 19,88 & 65,99 & 22,80 & 0,98 \\
Impresión general & 63,00 & 8,02 & 64,55 & 8,64 & $-0,59$
\end{tabular}

De hecho, al contrastar los promedios de clasificación por orientación sexual, se evidenciaron diferencias significativas entre las actitudes de todos los participantes hacia hombres homosexuales $(X=77,45, S D=23,71)$ y heterosexuales $(X=85,33, S D=12,38)(t(39)=2,35, p=0,02) ; y$ entre mujeres homosexuales $(X=77,25, S D=23,67)$ y heterosexuales $(X=87,60, S D=10,94)$ $(t(39)=2,97, p=0,00)$.
TABLA 2.

Actitud hacia grupos sociales según grupo de tratamiento Grupos sociales

\begin{tabular}{lllll}
\multicolumn{2}{l}{ GRUPO CONTROL } & \multicolumn{2}{l}{ GRUPO EXPERIMENTAL } \\
$\mathrm{M}$ & DE & $\mathrm{M}$ & $\mathrm{DE}$ & $\mathrm{t}$ \\
76,26 & 21,44 & 81,32 & 25,62 & $-0,68$ \\
82,35 & 15,27 & 88,31 & 7,95 & $-1,55$ \\
78,41 & 17,51 & 77,06 & 26,39 & 0,19 \\
80,67 & 14,78 & 85,17 & 12,06 & $-1,06$ \\
73,15 & 21,77 & 71,97 & 31,69 & 0,14 \\
77,17 & 18,06 & 83,35 & 13,96 & $-1,21$ \\
80,17 & 21,05 & 74,74 & 26,37 & 0,72 \\
81,88 & 17,45 & 83,95 & 16,56 & $-0,39$ \\
78,85 & 21,84 & 75,66 & 25,86 & 0,42 \\
81,99 & 17,04 & 85,56 & 13,98 & $-0,72$ \\
79,89 & 21,17 & 88,43 & 12,07 & $-1,57$ \\
66,36 & 26,39 & 75,94 & 22,57 & $-1,23$ \\
85,35 & 11,93 & 89,87 & 9,64 & $-1,32$ \\
78,22 & 21,11 & 82,51 & 16,36 & $-0,72$ \\
82,44 & 16,31 & 87,30 & 12,09 & $-1,07$ \\
78,88 & 14,87 & 82,08 & 13,93 & $-0,70$
\end{tabular}

\section{Discusión}

Considerando que las condiciones corporales provocadas por el ambiente tienen efectos sobre la formación de la percepción social (Bedia \& Castillo, 2010) se sometió a prueba una hipótesis derivada del estudio de Williams \& Bargh (2008), en donde se esperaba que las personas ubicadas en una sala a temperatura fría tuviesen actitudes más desfavorables hacia los grupos sociales que las que se situaban en una sala a temperatura ambiente.

Según los resultados obtenidos, se reconoce la falta de diferencias significativas entre grupos de tratamiento en la formación de impresiones y actitud hacia grupos sociales, es decir, los datos recolectados aportan evidencia contra la hipótesis planteada.

A pesar de ello, el grupo experimental reportó una sensación corporal de frío significativa, no prevista ni desagradable (t $(38)=3,075, p=0,004)$, elemento que además de ser deseado por los investigadores, evidencia que la manipulación de la variable fue efectiva. Por eso, es necesario el desarrollo de más pruebas que 
determinen el efecto de la temperatura ambiental en los procesos cognitivos, ya que desde otras investigaciones se ha demostrado la relación de la experiencia corporal con distintas manifestaciones de la cognición (Bargh \& Shalev, 2012, Williams \& Bargh, 2008).

Se considera que algunas de las razones que influyeron en la comprobación de la hipótesis, fueron que los grupos de tratamiento presentaron diferencias marginalmente significativas según sexo $(p=0.058)$, lo cual dificultó la comparación entre los mismos. Además, en el experimento no se incluyeron mediciones de la temperatura ambiental de la sala del grupo control, lo cual pudo influir en la varianza de condiciones.

La ausencia de los efectos de la temperatura también pudo deberse a fallas en la adherencia de los investigadores al protocolo o en la conformación de los reactivos de las escalas, hecho que recalca la importancia de examinar la validez del instrumento dentro del contexto de investigación y la homogeneidad de la conjetura de los encargados.

A pesar de lo anterior, es de interés recalcar que la calificación de los 40 participantes se diferenció en 8 puntos entre hombres homosexuales $(X=77,45)$ y heterosexuales $(X=85,33)$ y 10 puntos entre mujeres homosexuales $(X=77,25)$ y heterosexuales $(X=87,60)$. Datos que evidencian significancia estadística $(p=0.023$ y $p=0.005$, respectivamente) y que son indicio de homofobia en la muestra.

Estos resultados coinciden con los estudios de Banea, Calvente \& Díaz (2013), Beirute-Herrera et al. (2014) y Pinos \& Pinos (2011), quienes encontraron que adolescentes y jóvenes, poseen una actitud desfavorable hacia las personas homosexuales, a pesar de estar insertos en el sistema educativo. Siendo el nivel de cercanía con un miembro lésbico-gay, la orientación sexual (Pinos \& Pinos, 2011) y el género del participante (Banea et al, 2013) algunos de los elementos más importantes a la hora de emitir un criterio hacia estos grupos sociales.

Si bien no fue objetivo del estudio, cabe resaltar la trascendencia de incrementar la investigación sobre muestras de homofobia en la población universitaria, que aunque se encuentra en un ambiente enriquecido que promueve cambios culturales, aún manifiesta la presencia de prejuicios hacia la diversidad afectivo-sexual.

En conclusión, la manipulación de la variable independiente no generó diferencias significativas sobre los grupos de tratamiento. Lo cual, permite cuestionar el protocolo ejecutado, las escalas empleadas y la teoría misma del embodiment. Sin embargo, es relevante resaltar dos puntos, (a)las diferencias de calificación de la muestra hacia las personas homosexuales, las cuales evidencian diferencias estadísticas significativas; y (b)la necesidad de continuar con la investigación en la cognición corporizada y las muestras de homofobia en la población joven con altos grados de alfabetización.

\section{Notas}

1. Estudio elaborado para el curso PS1017. Procesos Psicológicos Básicos bajo la supervisión docente de Jorge Prado Calderón.

\section{Referencias}

Asch, S. (1952). Social psychology. New Jersey: Prentice Hall.

Banea, D., Calvente, M. \& Díaz, M. (2013). Homosexualidad: ¿Qué actitud tienen los universitarios al respecto? ReiDoCrea, 2, 130-137.

Bargh, J. A. \& Shalev, I. (2012). The substitutability of Physical and Social Warmth in Daily Life. American Psychological Association,12(1), 154-162.

Bedia, M. G. \& Castillo, O. L. (2010). Hacia una teoría de la mente corporizada: La influencia de los mecanismos sensomotores en el desarrollo de la cognición. Anfora, 17(28), 101-124

Beirute-Herrera, J., Angulo-Castro, V., Fallas-Gamboa, K. \& Prado-Calderón, J. (2014). Efecto del asco sobre las actitudes hacia hombres homosexules: evidencias de cognición corporizada en una muestra costarricense. Wimblu, 9, 7-21.

Corbeta, P. (2003). Social Research. Theory, Methods and Techniques. London: SAGE.

Creswell, J. (2012). Educational research: planning, conducting, and evaluating quantitative and qualitative research. 4th ed. Phoenix: Pearson.

Encabo, J. V. (2005). Mentes híbridas: cognición, representaciones externas y artefactos epistémicos. AIBR Revista de Antropología Iberoamericana, 1-36.

Fontes, S., García, C., Quintanilla, L., Rodríguez, R., Rubio, P. y Sarriá, E. (2015) Fundamentos de Investigación Psicológica. Madrid: Universidad Nacional Estatal a Distancia.

Inbar, Y., Pizarro, D. A. \& Bloom, P. (2012). Disgusting smells cause decreased liking of gay men. Emotion, 12(1), 23-27.

Letheby, C. (2012). In defence of embodied cognition: a reply to Fred Adams. Phenomenology and the Cognitive Sciences, 11(3), 403-414. 
Pinos, V. \& Pinos, G. (2011). Actitudes de los adolescentes escolarizados de Cuenca hacia la diversidad sexual. Maskana, 2(1), 1-16.

Rodríguez, R. D. (2012). Análisis de la Cognición Corporizada desde la perspectiva de la filosofía. (Tesis de Maestría, Universidad Autónoma del Estado de Morelos). Recuperado de http://www.academia.edu/1639656/An\%C3\%A1lisis de_la_Cognici\%C3\%B3n_Corporizada_desde_la_perspectiva_de_la_ filosof\%C3\%ADa

Soto, J. (2007). La cognición hecha cuerpo florece en metáforas En A. Ibañez, \& D. Cosmelli, (Ed.) Nuevos Enfoques de la Cognición, Acción e Intención(pp.71-90). Santiago: Universidad Diego Portales.

Wilson, R. \& Foglia, L. (2016). Embodied Cognition En The Stanford Encyclopedia of Philosophy. Recuperado de http://plato.stanford.edu/archives/spr2016/ entries/embodied-cognition/

Williams, L. E. \& Bargh, J. A. (2008). Experiencing physical warmth promotes interpersonal warmth. Science, 322, 606-607.

\section{Anexo}

UNIVERSIDAD DE COSTA RICA

FACULTAD DE CIENCIAS SOCIALES

ESCUELA DE PSICOLOGÍA

\section{CONSENTIMIENTO INFORMADO}

Actitudes de estudiantes universitarios hacia grupos sociales en el contexto costarricense.

Investigadores: Fiorella Jara Sanabria, Andrés Lizano Pérez y Monserrat Naranjo Fallas.

PROPÓSITO DEL PROYECTO:Mediante este proyecto se pretende explorar las actitudes de estudiantes universitarios hacia grupos sociales en el contexto costarricense.

¿QUE SE HARÁ?: Las y los participantes del estudio deberán trasladarse a un aula u oficina el día y la hora que se le asigne y contestar un cuestionario que contiene preguntas sociodemográficas y algunas escalas de actitudes. Se prevé que la participación tenga una duración de 15 minutos.

RIESGOS: Participar en esta actividad no significa ningún riesgo físico, psicológico o legal para usted. No obstante, si llegara a sentir incomodidad podrá comunicarle a la persona investigadora que no desea continuar participando.
BENEFICIOS:Como resultado de su participación en este estudio, no obtendrá ningún beneficio directo, sin embargo, es posible que las y los investigadores desarrollen un mayor conocimiento acerca de las actitudes hacia los grupos sociales.

Antes de dar su autorización para este estudio usted debe haber hablado con Fiorella Jara Sanabria, Andrés Lizano Pérez o Monserrat Naranjo Fallas y ellos deben haber contestado satisfactoriamente todas sus preguntas. Si quisiera mayor información puede obtenerla al 8503-5990. Cualquier consulta adicional puede comunicarse con el docente a cargo, Jorge Esteban Prado Calderón, al correo jorge.prado@ucr.ac.cr o al teléfono 8718-8223.

Recibirá una copia de esta fórmula para su uso personal.

Su participación en este estudio es voluntaria. Tiene el derecho de negarse a participar o a discontinuar su participación en cualquier momento, sin que esta decisión le perjudique.

Su participación en este estudio es confidencial, los resultados podrían aparecer en una publicación científica o ser divulgados en una reunión científica pero de una manera anónima.

Si está de acuerdo en participar, por favor complete el cuestionario que se le facilitará. Recuerde que su participación es voluntaria y confidencial. No se requiere su nombre para participar en esta investigación. 